\title{
Involvement of Neuropeptide $Y$ in Post-Incisional Nociception in Rats
}

\author{
Shivani Gupta Mayank Gautam Pranav Prasoon Rahul Kumar \\ Subrata Basu Ray Saroj Kaler Jhajhria \\ Departments of Anatomy, All India Institute of Medical Sciences, New Delhi, India
}

\section{Keywords}

Analgesia · Central nervous system · Intra-spinal administration · Neuropeptides · Opioids

\begin{abstract}
Background: Neuropeptide Y (NPY) is abundantly distributed in the mammalian nervous system. Its role in nociception arising from inflammatory and neuropathic pain conditions has been elucidated. However, its involvement in post-incisional nociception, particularly at the spinal cord level, is relatively unknown. Purpose: Management of postoperative pain is suboptimal. Evaluation of changes at the spinal level could facilitate better understanding of neural mechanisms underlying this type of pain. Methods: Rats were subjected to hind paw incision and spatiotemporal pattern of NPY expression in the dorsal horn was investigated by immunohistochemistry. Next, rats were implanted with intrathecal catheters using previously standardized procedure. NPY was injected into the intrathecal space by an indwelling catheter and behavioral assessment of nociception was performed. Results: Higher expression of NPY was observed in the superficial laminae of the dorsal horn. After incision, specific changes were observed like an abrupt decrease at $3 \mathrm{~h}$ after
\end{abstract}

incision, which could be correlated with the intense nociception at this time. In contrast to morphine administration, which attenuated all 3 behavioral parameters of nociception, NPY decreased guarding behavior and thermal hyperalgesia during the acute phase. Conclusions: NPY is extensively expressed in the superficial laminae of the spinal cord and exhibit marked changes after incision. Nociception is also decreased after its administration. Hence, it is likely involved in post-incisional nociception. This information could have clinical relevance.

(c) 2018 S. Karger AG, Basel

\section{Introduction}

Postoperative pain can delay an early discharge from hospitals following surgery [1]. Considering its magnitude, guidelines were framed by the American Pain Society in consultation with other professional bodies for effectively managing its occurrence [2]. Pain is alternately known as nociception in experimental animals and is evaluated by various reflexive or escape behaviors [3]. This is because pain is also subjective and its exact nature in animals remains unknown to us.

\section{KARGER}

(c) 2018 S. Karger AG, Basel

E-Mail karger@karger.com

www.karger.com/aon
Dr. Saroj Kaler Jhajhria

Pain Research Laboratory

Department of Anatomy, All India Institute of Medical Sciences

Ansari Nagar, New Delhi 110029 (India)

E-Mail drsarojkaler@ aiims.edu, sarojkaler@gmail.com 
Noxious sensation is transmitted to the spinal cord by a specific set of peripheral sensory nerve fibers called the $\mathrm{A}$-delta and $\mathrm{C}$ fibers. Their predominant sites of termination are the interneurons in Rexed's laminae I-II (superficial lamina) of the dorsal horn [4]. Further, these interneurons end on projection neurons situated in lamina I and $\mathrm{V}$ which transmit the noxious sensation to supraspinal centers like the thalamus and the lateral parabrachial nucleus. The propagation of pain from the spinal cord to higher neural centers is interrupted by spinal anesthesia, an extensively used procedure in hospitals for the relief of postoperative pain [5].

Various neurotransmitters and neuropeptides are present in the superficial lamina of the spinal cord, which not only mediate but also regulate synaptic activity. The major excitatory and inhibitory neurotransmitters are glutamate and GABA, respectively [6]. The projection neurons are glutamatergic whereas the interneurons can be either excitatory (glutamatergic) or inhibitory $(\gamma$-aminobutyric acid or glycine) in nature. GABAergic neurons have been shown to co-express neuropeptide $\mathrm{Y}$ (NPY), which is one of the abundantly distributed neuropeptides in the mammalian nervous system [7]. NPY receptors (Y1, Y2, Y4, Y5, and Y6) are G protein-coupled receptors. Among the receptors, the Y1 receptor likely mediates several of its actions [8]. It decreases intracellular $\mathrm{Ca} 2+$ concentration and inhibits adenylyl cyclase activity. The role of NPY in excessive food intake has been debated of late [9]. Besides, it regulates circadian rhythm, cardiovascular functions like blood pressure and heart rate, locomotion, and mood $[8,10]$. Evidence has also accumulated for its involvement in nociception. For example, Taylor et al. [11] have reported that direct intra-spinal administration of NPY attenuated acute inflammatory nociception in rats. Again, chronic pain arising from experimental nerve damage was also reduced by NPY [12]. However, its effect on postoperative pain has not been completely elucidated. For example, Yalamuri et al. [13] reported that intrathecal administration of NPY in rats attenuated guarding behavior, which is an indicator of spontaneously occurring postoperative pain. However, expression of NPY in the spinal cord during the post-incision period was not studied. This could be important as definitive changes in NPY immunoreactivity was observed in specific sectors of the dorsal horn of the spinal cord after induction of neuropathic pain [14]. Consequently, the main objective of the present work was to characterize the temporospatial changes in the expression of NPY in the spinal cord of rats and to correlate this with post-incisional nociception. The effect of intrathecal administration of
NPY on nociception was also determined and compared to morphine. The "Hind paw incision" model, standardized by Brennan et al. [15] was used for the study.

\section{Materials and Methods}

\section{Animals and Drug Administration}

Male Sprague-Dawley rats (275-325 g; $n=66)$ were procured from experimental animal facility of AIIMS, New Delhi, after receiving permission from the Institute Ethics Committee (762/ IAEC/13 and 903/IAEC/15). Rats were housed in cages with freely available food and water. Experimental procedure were divided into 2 separate parts: (1) immunohistochemical localization of NPY in the spinal cord $(n=48)$ and (2) behavioral assays for nociception after intra-spinal administration of morphine or NPY $(n=18)$. Both groups were subjected to paw incision except for control animals in the first part of the study. Baseline values for nociceptive tests were obtained for the concerned rats before proceeding with the incision. Intra-spinal drug administration was done by a chronically implanted intrathecal catheter. Control rats received $0.9 \%$ saline $(10 \mu \mathrm{L})$ whereas drug-treated rats received either NPY (Tocris Bioscience, UK) or morphine $(30 \mu \mathrm{g} / 10 \mu \mathrm{L})$. The latter was purchased from a government-approved pharmacy. Drugs were administered only once during the experiment, $15 \mathrm{~min}$ prior to the paw incision.

\section{Hind Paw Incision}

Procedure of incision has been reported previously [16]. Briefly, rats were anaesthetized with inhalation of isoflurane and the right hind paw taken out through an opening in a sterile drape. The plantar aspect was swabbed with povidone-iodine solution and isopropyl alcohol. A $1 \mathrm{~cm}$ long midline incision was made on the skin using a no. Eleven scalpel blade. The deep fascia was cut to expose the flexor digitorum brevis muscle. The muscle was also incised along its long axis for $1 / 2$ without disturbing the origin or insertion. A forceps was inserted into the cut and the limbs of the forceps slightly separated. Thereafter, skin was opposed with silk $\left(4-0\right.$, Ethicon $\left.{ }^{\circledR}\right)$ using 2 mattress sutures. Neosporin ointment was applied and the rats transferred to a warm recovery chamber. Later, they were returned to their home cages containing clean bedding (Alpha-dri, Shepherd Speciality papers, Milford, NJ, USA) for preventing further damage to the injured paw. Control rats were anesthetized without paw incision.

\section{Immunohistochemical Localization of NPY}

Rats $(n=48)$ were deeply anaesthetized with phenobarbitone injection (100 mg/kg i.p). Intracardiac perfusion was initially performed with cold $0.1 \mathrm{M}$ phosphate buffer saline (PBS) followed by $4 \%$ paraformaldehyde in PBS (fixative solution) using a perfusion pump. The skin of the back was incised followed by laminectomy of the T10 to L3 vertebrae in order to expose the spinal cord. The 13 th thoracic vertebra was identified by the last pair of floating ribs. The part corresponding to lumbar 4-5 spinal segments (present at the T13-L1 level) was isolated and kept in fixative solution for 3 days. Cryo-protection was done by immersing in 15 and then $30 \%$ sucrose solution. Finally, transverse sections $(20 \mu \mathrm{m})$ were cut in a cryostat (Leica CM 1950; Germany) and collected in multivial cul- 
ture plates containing PBS solution. The sections were stored at $-20^{\circ} \mathrm{C}$. On the day of staining, 10 sections/animal were processed for immunoperoxidase staining. Endogenous peroxidase activity was quenched using $0.3 \%$ hydrogen peroxide in $80 \%$ methanol for $30 \mathrm{~min}$. Nonspecific binding was blocked by $10 \%$ normal goat serum containing Triton-X 100. The sections were incubated with primary antibody for NPY (1:1,000; rabbit polyclonal; Abcam, UK) for $48 \mathrm{~h}$ at $4{ }^{\circ} \mathrm{C}$. According to the manufacturer's data sheet, the peptide cross-reacts with pancreatic polypeptide found in the gut but not with other peptides like somatostatin and substance P (SP). The sections were then incubated with biotinylated secondary antibody and Avidin-Biotin complex in succession for $2 \mathrm{~h}$ each (Vector labs, Burlingame, CA, USA). Visualization of the immune complex was done by $0.05 \%$ diaminobenzidine tetrahydrochloride in PBS in the dark. Thereafter, sections were taken on gelatin coated slides, dehydrated, cleared, and mounted using DPX. Images were captured under a light microscope (Nikon 80i). Nonspecific staining was assessed using the same procedure except that the primary antibody was not added. Few of the sections were stained with $1 \%$ Cresyl violet for visualizing neurons in the various Rexed's laminae.

\section{Estimation of Nociception}

Nociceptive assessment included both non-evoked (guarding) and evoked - mechanical allodynia (nociceptive response to a nonnociceptive stimulus) and thermal hyperalgesia (exaggerated response to a nociceptive stimulus) parameters. These were determined up to day 4 after incision for non-evoked and day 7 for evoked nociception $[15,16]$.

\section{Guarding Behavior}

The incised paw was instinctively protected by the animal after incision. Briefly, rats were placed over a wire mesh platform and covered by plastic enclosures $(16 \times 16 \times 16 \mathrm{~cm}$ size $)$ for $30 \mathrm{~min}$ for acclimatization. Following this, the position of the hind paws was observed from below the mesh for $1 \mathrm{~h}$ ( 12 bins of 5 min each). Scoring was performed according to whether the incised paw was off the mesh (2), on the mesh but without weight transmission (1), and firmly pressed along with weight transmission (0). Scoring was done during the first $1 \mathrm{~min}$ of every $5 \mathrm{~min}$ bins. Final values (between 0 and 24) were the cumulative pain score and estimated by deducting the score of the normal paw from the incised paw. A higher value meant an increase in nociceptive behavior.

\section{Mechanical Allodynia}

Allodynia was evaluated by the "Up-down method". Rats were allowed to remain over the wire mesh platform, which had $8 \times 8$ $\mathrm{mm}$ gaps. A series of fine nylon monofilaments of different sizes (3.61, 3.84, 4.08, 4.31, 4.56, 4.74, 4.93, and 5.18) called von Frey filaments (North Coast Medical Inc. San Jose, CA, USA) were used for determining nociception. These exerted a predefined amount of pressure $(0.4-15 \mathrm{~g})$. Filaments were inserted in series through gaps in the mesh. The tip was allowed to touch the peri-incisional area, medial to the incision, as has been reported previously, and firm pressure was exerted [15]. This caused the filament to buckle. The end point was the reflex withdrawal of the foot. In case of a response, the next lower filament was used. A higher size filament was used if no response was noted. Under basal conditions, rats do not withdraw their paw to the highest pressure $(15 \mathrm{~g})$. The $50 \%$ withdrawal threshold (g) was calculated using a specific algorithm [17]. Lower values indicate more nociception.

\section{Thermal Hyperalgesia}

Rats were placed over a special glass platform and covered with perspex enclosures (Plantar test apparatus, UGO Basile, Italy). The acclimatization period was $15 \mathrm{~min}$. An infrared source focuses a thermal stimulus from below the platform at the incision site. Under basal condition, there is reflex withdrawal of the foot between $8-10 \mathrm{~s}$. Cut-off latency value was $20 \mathrm{~s}$ to prevent damage to the paw. A motion sensor detects the movement of the paw and cut-off the beam. The time period (withdrawal latency), which is automatically detected, is reduced after incision. Percentage maximum possible effect (\% MPE) was derived from the formula: ([drug induced latency-baseline latency]/[cutoff latency-baseline latency] $) \times 100$. Derivation of $\%$ MPE from raw latency values has several advantages like values are normalized for comparison [18].

\section{Intrathecal Implantation of Catheter}

The procedure for implantation of intrathecal catheter has been reported previously [19]. Under isoflurane anesthesia, the rats were placed in a stereotaxic apparatus. The skin on the back of the neck was incised and the muscles retracted to expose the cisternal membrane. This was incised and a PE-5 size catheter was inserted into the intrathecal space. It was advanced in a caudal direction till it reached the upper part of the lumbar enlargement of the spinal cord $(\sim 8.5 \mathrm{~cm}$ from the cut). The skin was sutured. The remaining part of the catheter was placed externally for drug administration, using a $30-\mathrm{G}$ needle fixed to a Hamilton syringe. The rats were allowed to recover for 5 days before starting the experimental work.

\section{Statistical Analysis}

Data were analysed by GraphPad Prism, version 5 (GraphPad software La Jolla, CA, USA). Values were expressed as means \pm SEM. These were analysed by 1 -way analysis of variance followed by Bonferroni multiple comparison test. Assuming nonparametric distribution of values of withdrawal threshold for estimating allodynia, Kruskal-Wallis test followed by Dunn's multiple comparison was used. $p<0.05$ was considered statistically significant.

\section{Results}

\section{NPY Expression in the Superficial Laminae of the Spinal Cord}

Compared to cresyl-violet stained sections, where abundant profiles of neuronal cell bodies containing Nissl substance were observed in the superficial laminae, numerous punctate varicosities, stained for NPY, were observed (Fig. 1). At many instances, these were arranged in a linear fashion and appeared to be presynaptic axonal terminals. Some of these varicosities were arranged in close proximity to neuronal cell bodies in the periphery. In contrast to the superficial laminae, neuronal cell bodies, stained for NPY, was observed in the deeper part of the dorsal horn.

Subsequently, changes in the spatiotemporal pattern of expression of NPY in the superficial laminae were corre- 

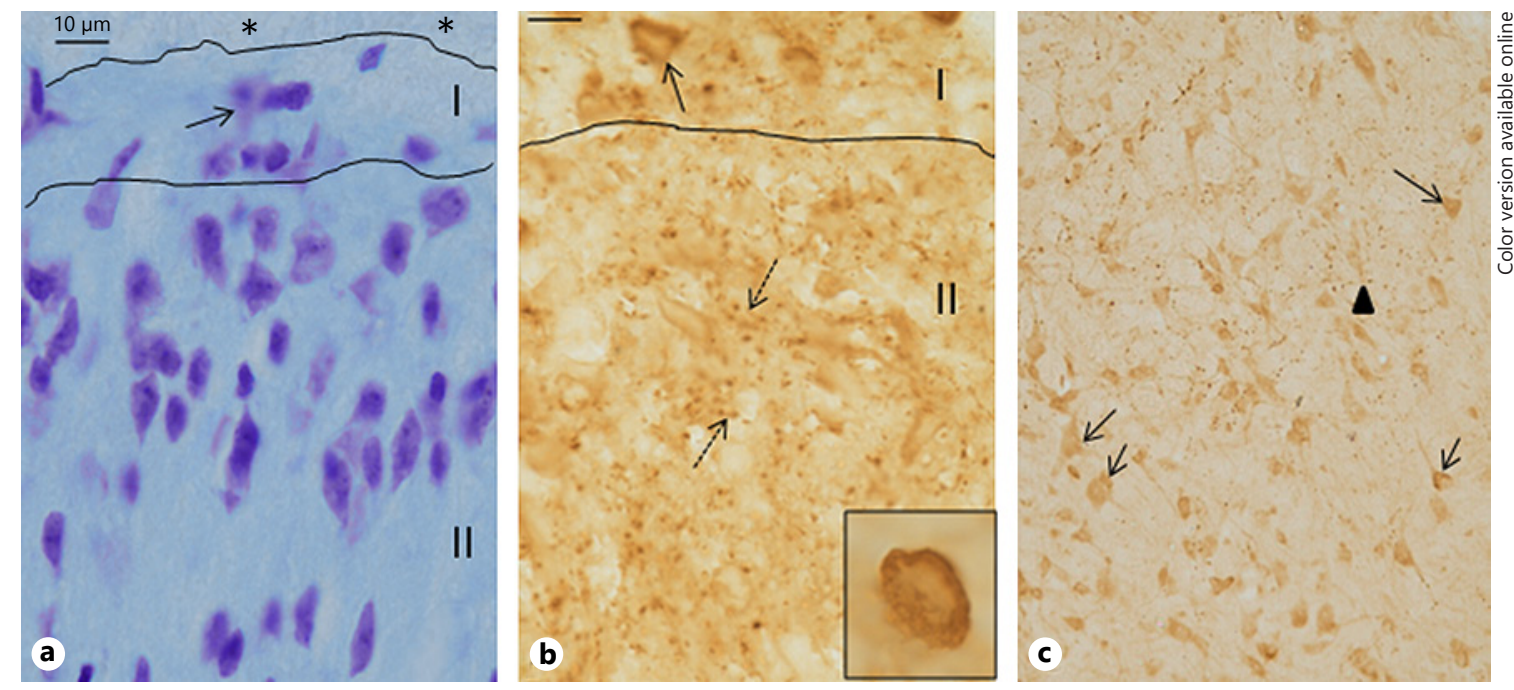

Fig. 1. a-c Compared to Nissl stained spinal cord sections (a), where neuronal cell bodies can be identified in mainly horizontal (lamina I) and radial arrangement (lamina II), NPY immunoreactivity is observed as punctate varicosities in Rexed's laminae I-II. b Some are arranged in a row whereas others are in an irregular circle (broken arrows) and could indicate synaptic junctions. A neuronal cell body can be seen in lamina I, densely covered with these varicosities (also shown in inset). In deeper laminae III-IV (c), both varicosities (arrowhead) as well as immunostained cell bodies (arrows) can be observed. * Tract of Lissauer. Bar represents $10 \mu \mathrm{m}$. lated with different time periods after incision (Fig. 2, 3). In the control group, expression of NPY was present over the entire dorsal horn, although higher expression was observed over the superficial laminae, when compared to Nissl-stained sections (Fig. 2). Within an hour after incision, staining intensity for NPY had started decreasing. The varicosities were fewer in number. Further decrease was noted at $3 \mathrm{~h}$, which exhibited a "washed-out" appearance. Thereafter, there was a slight increase in NPY staining at $6 \mathrm{~h}$ and then again at $12 \mathrm{~h}$. Surprisingly, staining intensity decreased on day 1 , which was somewhat similar to that observed at $3 \mathrm{~h}$ (Fig. 3). The immunostaining slightly increased at day 3 and a further increase was observed at day 5 after incision, when its appearance was qualitatively similar to the control group with prolific increase in varicosities. Nonspecific staining was absent from tissue sections, not exposed to the primary antibody (data not shown).

\section{Behavioral Assessment of Nociception}

Paw incision led to maximum nociception between 2 and $8 \mathrm{~h}$ (Fig. 4). Thereafter, this gradually decreased though the various behavioral parameters did not attain basal levels by the end of the observation period. Compared to saline, both NPY and morphine significantly attenuated cumulative pain score between $2 \mathrm{~h}$ - day 2 (for example, at $2 \mathrm{~h}$, saline was $19.83 \pm 0.87$; morphine was $6.16 \pm 1.6$ whereas NPY was $5.2 \pm 1.7$ ). Moreover, the pain scores of both morphine and NPY treated groups but not saline showed baseline values at day 4 . The maximum allodynia (nociception) was at $2 \mathrm{~h}(0.4 \mathrm{~g})$ as evident from the extremely low value of $50 \%$ withdrawal threshold. Lower threshold values indicate increased nociception. Withdrawal thresholds increased gradually up to day 7 . Morphine alone increased withdrawal threshold (reduced allodynia $)$ at $2 \mathrm{~h}(7.9 \pm 1.8 \mathrm{~g})$ in comparison to both saline and NPY treated groups. Allodynia was also significantly inhibited toward the end of the observation period (days 5-6) by both morphine and NPY. Additionally, morphine reduced allodynia in relation to NPY on day 7 though not in reference to saline; \% MPE was decreased by both NPY $(56.33 \pm 0.67)$ and morphine $(46.33 \pm 9.04)$ compared to saline $(89.83 \pm 5.7)$ although at $2 \mathrm{~h}$, morphine had a greater anti-nociceptive effect than NPY. Subsequently, both NPY and morphine significantly reduced thermal hyperalgesia up to day 2. Overall antinociceptive effect of both NPY and morphine was also evaluated by comparison of values of area under curve ( $\triangle$ AUC; Fig. 5). Both NPY (29.5 \pm 4.8$)$ and morphine $(35.77 \pm 2.6)$ decreased guarding behavior significantly compared to saline treatment $(80.38 \pm 2.4)$. Regarding allodynia, morphine $(68.98 \pm 3.6)$ and not NPY $(53.02 \pm$ 3.7) alone had a significant effect compared to saline $(39.93 \pm 3.4)$. However, both morphine and NPY decreased thermal hyperalgesia significantly. 

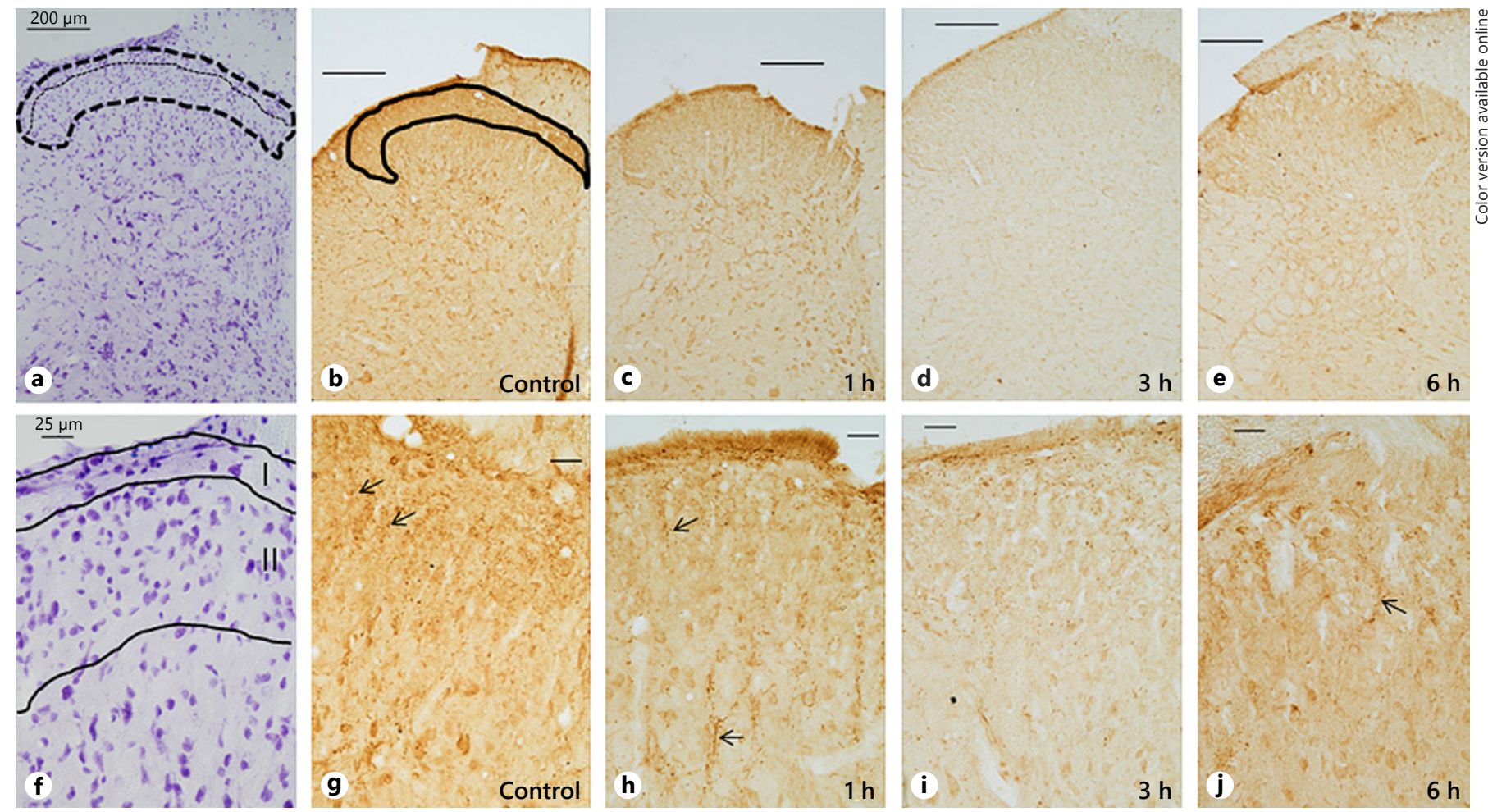

Fig. 2. a-j Spatio-temporal pattern of NPY expression in the dorsal horn before and after incision under low (upper panel) and high (lower panel) magnification (b-e, g-j). Corresponding Nissl stained section for delineation of Rexed's laminae I-II (a, f). NPY was expressed in the entire dorsal horn in the control group but higher level of expression was noted in lamina I and outer part of

\section{Discussion}

The study shows that NPY is predominantly expressed by nerve fibers in the superficial laminae of the dorsal horn of the spinal cord. Higher expression was observed over lamina I and outer part of lamina II, which are key areas receiving afferent noxious stimuli from the periphery. Similarly, a dense network of NPY immunoreactive fibers was noted earlier in the superficial laminae [20]. Few immunopositive neurons were also observed in lamina II in this earlier report though these were only observed in the deeper laminae in our work. A later study also showed higher expression of nerve terminals and varicosities in the superficial laminae, which were not affected by either dorsal rhizotomy or lesion in the dorsolateral fasciculus [21]. The authors concluded that the fibers were derived from interneurons in the dorsal horn. Furthermore, these neurons co-express GABA and thus are inhibitory in nature $[7,22]$. Selective expression of other neuropeptides associated with nociception like SP

lamina II (outlined area in b), mainly in the form of varicosities (arrows in $\mathbf{g}$ ). $1 \mathrm{~h}$ post-incision, expression had decreased $(\mathbf{c}, \mathbf{h}$ ) and further decrease was noted at $3 \mathrm{~h}(\mathbf{d}, \mathbf{i})$. An increase was present at $6 \mathrm{~h}(\mathbf{e}, \mathbf{j})$. Bars in upper and lower panels represent $200 \mu \mathrm{m}$ and $25 \mu \mathrm{m}$ respectively.

and calcitonin gene-related peptide were also noted in these laminae [23, 24]. Even $\mu$-opioid receptors, the opioid receptor subtype binding to morphine are also noted in this region [25]. Thus, the neural network has the mechanism for both propagating noxious stimuli to supraspinal areas (SP, calcitonin gene-related peptide) as well as for modulating this transmission (NPY, $\mu$ receptor). Since neuropeptides are generally synthesized only in the cell bodies, their absence from cell bodies at this site could imply that the NPY is derived from the abundant NPY immunopositive cell bodies in the deeper parts of the dorsal horn. Neurons, classically termed "antenna cells", localized in laminae IV-V, send their axons to more superficial laminae [26]. It might also be presumed that there could be rapid turnover of NPY in neurons of the superficial laminae with these neuropeptide being quickly transferred to the axons following synthesis. Administration of colchicine, which increases the aggregation of neuropeptides in cell bodies, was utilized for examining these neurons [27]. A detailed examination of neurons 

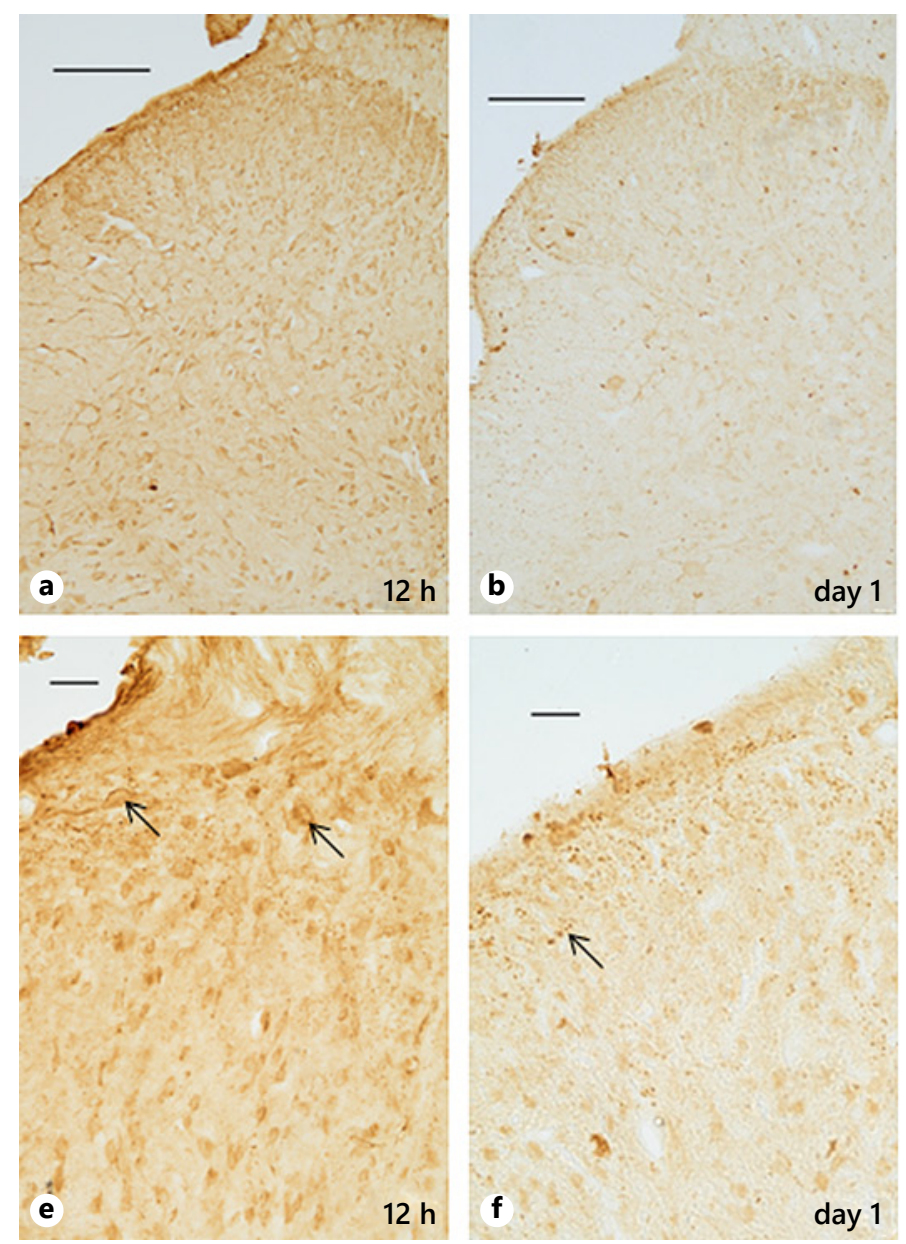

Fig. 3. a-h NPY immunostaining after incision under low (upper panel) and high (lower panel) magnification (a-d, e-h). Immunostaining continued to increase between 6-12 h. Punctate varicosities were observed around neuronal cell bodies, particularly in

within laminae I-III revealed that $15 \%$ of the inhibitory interneurons express NPY [28]. What could be the functional significance of expression of NPY? Data from several studies, including experiments using doxycycline-induced knockdown of NPY, convincingly showed that NPY could act as an "endogenous braking mechanism" and suppress transmission of noxious stimuli at the spinal cord level [29-31].

The initial decrease of NPY immunostaining between 1 and $3 \mathrm{~h}$ after incision, when nociception was at its highest level, can be correlated with the release of NPY from synaptic terminals as well as extrasynaptic sites [32]. This could modulate the transmission of pain signals. Neuropeptides are inactivated by extracellular peptidases upon release apart from binding to their cognate receptors because in contrast to small molecule neu-
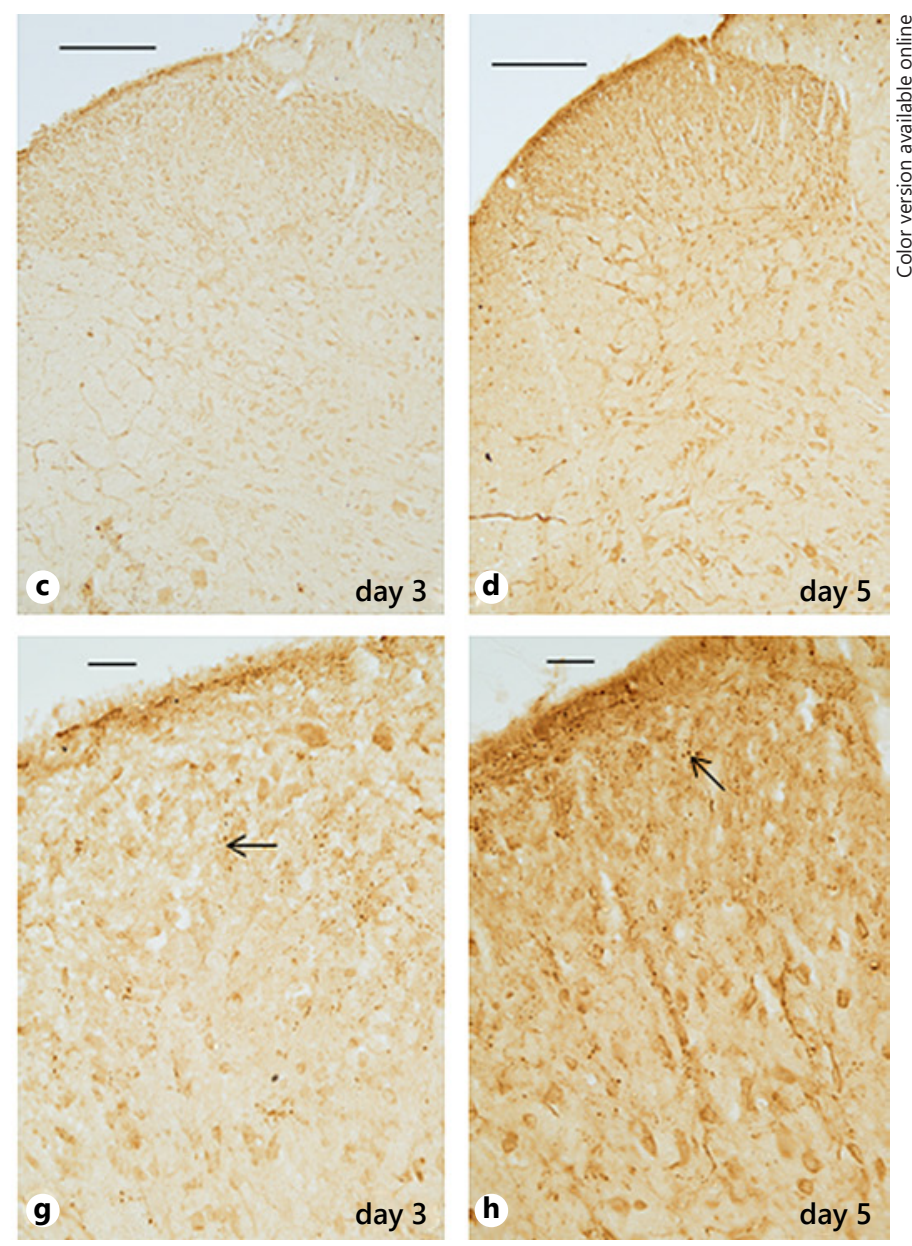

the superficial laminae I-II (arrows) at every time point. This was followed by decreased staining at day 1 . Subsequently, there was increase at day 3 and a further increase at day 5. Bars in upper and lower panels represent $200 \mu \mathrm{m}$ and $25 \mu \mathrm{m}$ respectively.

rotransmitters, there are no reuptake mechanisms for the former [32,33]. An increase, thereafter, between 6 and $12 \mathrm{~h}$ was likely the result of re-synthesis of this neuropeptide. However, neuropeptides are initially synthesized in precursor forms like Prepro-NPY, which are unsuitable for release, despite persistent volleys of action potentials. Incidentally, these precursor forms are recognized by polyclonal antibodies [23]. Restoration of the NPY level was interrupted on day 1, when its expression decreased again. Afterwards, there was a progressive increase in NPY expression up to day 5 when it was similar to the basal levels seen in naïve rats. However, nociception had still not normalized to pre-incision levels at this time interval. Compared to the release of small molecule neurotransmitters, release of neuropeptides require greater amount of neuronal excitation [32]. 


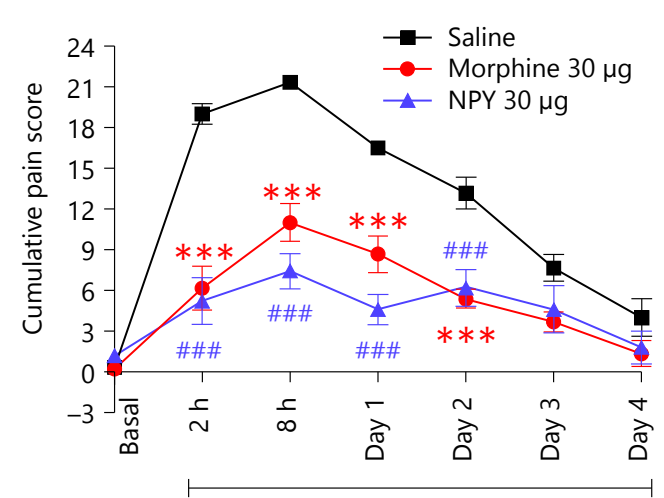

a

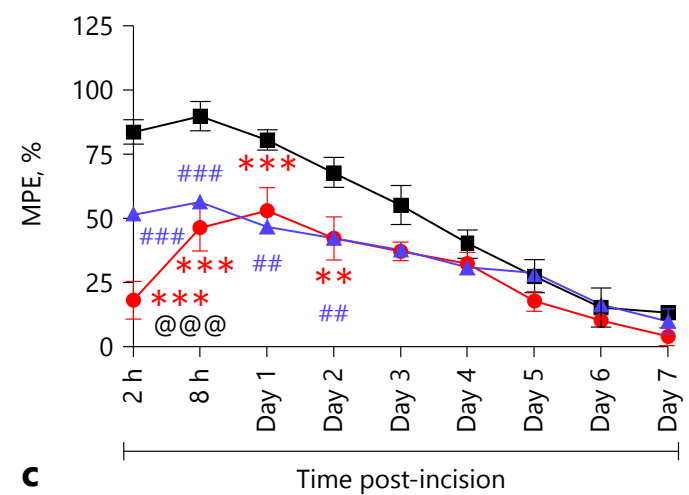

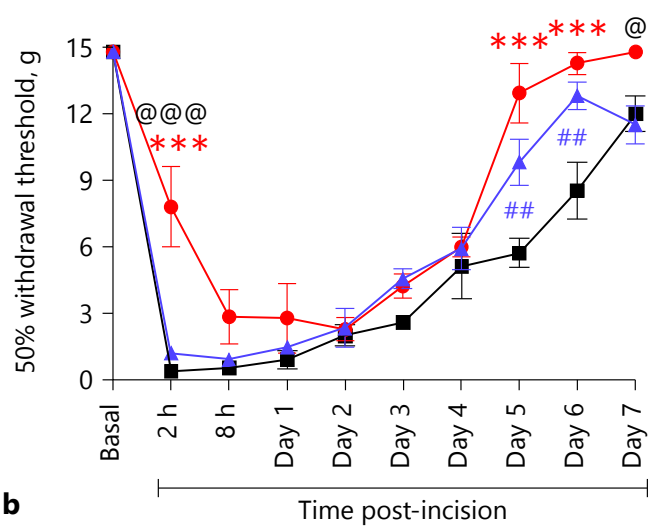

Fig. 4. a-c Antinociceptive effect of NPY after paw incision and its comparison with saline and morphine. Drugs were administered by intrathecal catheter. Compared to saline, NPY attenuated cumulative pain score (guarding; a) and \% MPE (thermal hyperalgesia; c) between $2 \mathrm{~h}$ to day 2 after incision $\left({ }^{\# \#}\right)$. Similar findings were noted with morphine $\left({ }^{* * *}\right)$. Statistically significant difference between NPY and morphine was not observed except at $2 \mathrm{~h}$, when $\%$ MPE values of morphine were significantly lower than NPY
(@@@). Allodynia (b), represented as 50\% withdrawal threshold (higher values indicate less nociception), was only attenuated by morphine at $2 \mathrm{~h}$ (acute phase) compared to both saline (***) and NPY (@@@). Later, morphine and NPY attenuated allodynia between days 5-6 compared to saline. On day 7, morphine was more effective than NPY ( $\left.{ }^{@}\right) .{ }^{@} p<0.05,{ }^{\#, * *} p<0.01, * * *$,\#\#\# $p<0.001$. NPY, neuropeptide Y; MPE, maximum possible effect.

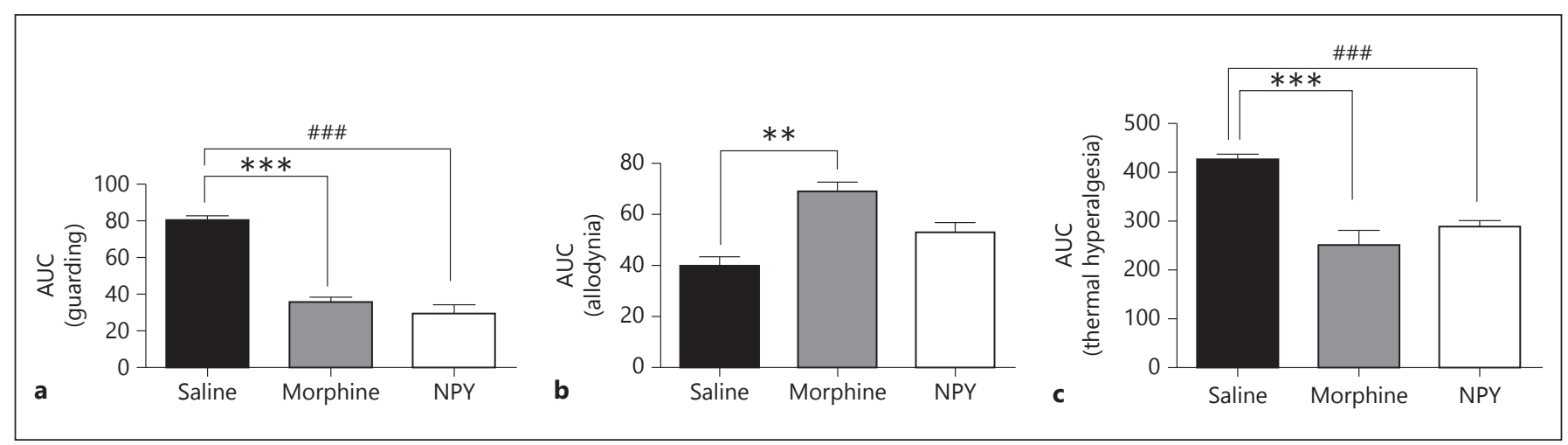

Fig. 5. a-c Overall antinociceptive effect over the entire observation period is represented as area under curve $(\triangle \mathrm{AUC})$. Values of both NPY and morphine were significantly different than saline for guarding and thermal hyperalgesia whereas morphine alone pro- duced significant difference than saline for allodynia. Interestingly, NPY and morphine did not differ in any of the behavior tests for nociception. ${ }^{* *} p<0.01,{ }^{* * *}$, \#\#\# $p<0.001$. NPY, neuropeptide Y. 
Thus, neuronal activity, immediately after incision, would be more prolific than at day 5 after incision is understandable. Alteration in SP expression in the superficial laminae has been previously reported after paw incision [23]. Again, Ji et al. [20] had reported an increase in NPY immunoreactivity in lamina II after peripheral inflammation, localized to the medial part of the dorsal horn. However, this was not observed in the current investigation. It might be due to the different animal models used for the respective studies (complete Freund's adjuvant-induced inflammation versus surgical incision). Changes in NPY expression also occurs in neuropathic pain though this is mainly localized in the deeper laminae (Rexed's laminae III-IV), which is in accordance with changes in the large diameter dorsal root ganglion neurons instead of the smaller sized cell soma observed after inflammatory pain [14].

Paw incision-induced nociception was comprehensively attenuated up to day 2 by intrathecal administration of morphine, considered to be the gold standard reference drug for the treatment of pain in patients [34]. However, allodynia was reduced only at $2 \mathrm{~h}$. It is well established that this anti-nociceptive effect is produced by binding to the $\mu$-opioid receptors in the superficial laminae of the dorsal horn [25]. NPY produced an equivalent relief of guarding behavior though its effect on thermal hyperalgesia was marginally less than morphine though significantly more than saline treated group. However, at $2 \mathrm{~h}$ alone after incision, morphine produced significantly greater anti-nociceptive effect than NPY. Allodynia was unaffected during the initial part of the observation period after NPY in contrast to morphine. This difference did not reach statistical significance between morphine and NPY when values for $\triangle A U C$ were compared. Overall, NPY appears to be comparable to morphine in its antinociceptive effect. Taiwo and Taylor [30], using a similar dose of NPY $(30 \mu \mathrm{g})$, also noted an antinociceptive effect of NPY on the Carrageenan-induced thermal hyperalgesia in the rat. The authors also reported that the Y1 recep- tor in the dorsal horn was responsible for this antinociceptive effect. No perceptible toxicity was observed after NPY administration in our study as in this earlier study [30].

In conclusion, the results of this study show a prominent involvement of NPY in postoperative pain in rats, both with reference to changes in its expression in the spinal cord following incision and the relief of nociception following drug administration. These findings could have clinical significance considering that NPY is an endogenous bioactive molecule and detailed safety studies with regard to biocompatibility would not be required.

\section{Acknowledgments}

Financial support was provided by Department of Biotechnology, Ministry of Science and Technology, Government of India (BT/PR14279Med/30/452/2010), from 2011 to 2014. Additional support by Prof. T.S. Roy, Prof. and Head, Department of Anatomy, AIIMS, New Delhi, in the latter part of the study is gratefully acknowledged. Laboratory assistance from Mr. B.K. Gayakwar, technical officer, and Mr. Rampravesh Manjhi, animal attendant, is also acknowledged.

\section{Disclosure Statement}

The authors have no conflict of interest to declare.

\section{Funding Sources}

Department of Biotechnology, Government of India.

\section{Author Contribution}

S.K.J. and S.B.R. devised the experimental protocol, analysed the data, and wrote the manuscript, S.G. and P.P. performed NPY localization, M.G. did the part on intra-spinal administration of NPY along with R.K.

\section{References}

1 Rae A: Reasons for delayed patient discharge following day surgery: a literature review. Nurs Stand 2016;31:42-51.

2 Chou R, Gordon DB, de Leon-Casasola OA, Rosenberg JM, Bickler S, Brennan T, Carter T, Cassidy CL, Chittenden EH, Degenhardt E, Griffith S, Manworren R, McCarberg B, Montgomery R, Murphy J, Perkal MF, Suresh S, Sluka K, Strassels S, Thirlby R, Viscusi E,
Walco GA, Warner L, Weisman SJ, Wu CL: Management of postoperative pain: a clinical practice guideline from the American pain society, the American society of regional anesthesia and pain medicine, and the American society of anesthesiologists' committee on regional anesthesia, executive committee, and administrative council. J Pain 2016;17:131157.
3 Le Bars D, Gozariu M, Cadden SW: Animal models of nociception. Pharmacol Rev 2001; 53:597-652.

4 Todd AJ: Neuronal circuitry for pain processing in the dorsal horn. Nat Rev Neurosci 2010; 11:823-836.

5 Chibueze CE, et al: Spinal anaesthesia drugs for caesarean section. Cochrane Database Syst Rev 2016;4:CD012134. 
6 Gangadharan V, Kuner R: Unravelling spinal circuits of pain and mechanical allodynia. Neuron 2015;87:673-675.

7 Laing I, Todd AJ, Heizmann CW, Schmidt $\mathrm{HH}$ : Subpopulations of GABAergic neurons in laminae I-III of rat spinal dorsal horn defined by coexistence with classical transmitters, peptides, nitric oxide synthase or parvalbumin. Neuroscience 1994;61:123-132.

8 Tatemoto K, Neuropeptide Y: History and overview; in Michel MC (ed): Neuropeptide Y and Related Peptides. Handbook of Experimental Pharmacology. Berlin, Springer, 2004, vol 162, pp 1-21.

9 Klockars A, Levine AS, Olszewski PK: Hypothalamic integration of the endocrine signaling related to food Intake. Curr Top Behav Neurosci 2018, Epub ahead of print.

10 Sayed S, Van Dam NT, Horn SR, Kautz MM, Parides M, Costi S, Collins KA, Iacoviello B, Iosifescu DV, Mathé AA, Southwick SM, Feder A, Charney DS, Murrough JW: A randomized dose-ranging study of neuropeptide $\mathrm{Y}$ in patients with posttraumatic stress disorder. Int J Neuropsychopharmacol 2018;21:3-11.

11 Taylor BK, Fu W, Kuphal KE, Stiller CO, Winter MK, Chen W, Corder GF, Urban JH, McCarson KE, Marvizon JC: Inflammation enhances $\mathrm{Y} 1$ receptor signaling, neuropeptide Y-mediated inhibition of hyperalgesia, and substance $\mathrm{P}$ release from primary afferent neurons. Neuroscience 2014;256:178-194.

12 Intondi $A B$, Dahlgren MN, Eilers MA, Taylor BK: Intrathecal neuropeptide Y reduces behavioral and molecular markers of inflammatory or neuropathic pain. Pain 2008; $137: 352-$ 365.

13 Yalamuri SM, Brennan TJ, Spofford CM: Neuropeptide $\mathrm{Y}$ is analgesic in rats after plantar incision. Eur J Pharmacol 2013;698:206212.

14 Intondi $\mathrm{AB}$, Zadina JE, Zhang X, Taylor BK: Topography and time course of changes in spinal neuropeptide $\mathrm{Y}$ immunoreactivity af- ter spared nerve injury. Neuroscience 2010; 165:914-922.

15 Brennan TJ, Vandermeulen EP, Gebhart GF: Characterization of a rat model of incisional pain. Pain 1996;64:493-501.

16 Spofford CM, Ashmawi H, Subieta A, Buevich F, Moses A, Baker M, Brennan TJ: Ketoprofen produces modality-specific inhibition of pain behaviors in rats after plantar incision. Anesth Analg 2009;109:1992-1999.

17 Chaplan SR, Bach FW, Pogrel JW, Chung JM, Yaksh TL: Quantitative assessment of tactile allodynia in the rat paw. J Neurosci Methods. 1994;53:55-63.

18 Allen JW, Yaksh TL: Assessment of acute thermal nociception in laboratory animals; in Luo ZD (ed): Pain Research: Methods and Protocols. NJ, Humana Press, 2004, pp 11-23.

19 Malkmus SA, Yaksh TL: Intrathecal catheterization and drug delivery in the rat; in Luo ZD (ed): Pain Research: Methods and Protocols. NJ, Humana Press, 2004, pp 109-121.

20 Ji RR, Zhang X, Wiesenfeld-Hallin Z, Hokfelt T: Expression of neuropeptide $\mathrm{Y}$ and neuropeptide $\mathrm{Y}(\mathrm{Y} 1)$ receptor mRNA in rat spinal cord and dorsal root ganglia following peripheral tissue inflammation. J Neurosci 1994; 14:6423-6434.

21 Ossipov MH, Zhang ET, Carvajal C, Gardell L, Quirion R, Dumont Y, Lai J, Porreca F: Selective mediation of nerve injury-induced tactile hypersensitivity by neuropeptide Y. J Neurosci. 2002;22:9858-9867.

22 Polgár E, Sardella TC, Watanabe M, Todd AJ: Quantitative study of NPY-expressing GABAergic neurons and axons in rat spinal dorsal horn. J Comp Neurol 2011;519:1007-1023.

23 Gautam M, Prasoon P, Kumar R, Reeta KH, Kaler S, Ray SB: Role of neurokinin type 1 receptor in nociception at the periphery and the spinal level in the rat. Spinal Cord 2016;54: 172-182.

24 Kaler S, Gupta S, Prasoon P, Gautam M, Ray SB: Role of calcitonin gene-related peptide in nociception resulting from hind paw incision in rats. J Anat Soc India 2017;66:7-14.

25 Ray SB, Gupta H, Gupta YK: Up-regulation of mu-opioid receptors in the spinal cord of morphine-tolerant rats. J Biosci 2004;29:51-56.

26 Szentagothai J: Neuronal and synaptic arrangement in the substantia gelatinosa rolandi. J Comp Neurol 1964;122:219-239.

27 Sasek CA, Elde RP: Distribution of neuropeptide Y-like immunoreactivity and its relationship to FMRF-amide-like immunoreactivity in the sixth lumbar and first sacral spinal cord segments of the rat. J Neurosci 1985;5:17291739.

28 Iwagaki N, Ganley RP, Dickie AC, Polgár E, Hughes DI, Del Rio P, Revina Y, Watanabe M, Todd AJ, Riddell JS: A combined electrophysiological and morphological study of neuropeptide $\mathrm{Y}$-expressing inhibitory interneurons in the spinal dorsal horn of the mouse. Pain 2016;157:598-612.

29 Mahinda TB, Taylor BK: Intrathecal neuropeptide $\mathrm{Y}$ inhibits behavioral and cardiovascular responses to noxious inflammatory stimuli in awake rats. Physiol Behav 2004;80: 703-711.

30 Taiwo OB, Taylor BK: Antihyperalgesic effects of intrathecal neuropeptide $Y$ during inflammation are mediated by Y1 receptors. Pain 2002;96:353-363.

31 Solway B, Bose SC, Corder G, Donahue RR, Taylor BK: Tonic inhibition of chronic pain by neuropeptide Y. Proc Natl Acad Sci U S A. 2011;108:7224-7229.

32 Van den Pol AN: Neuropeptide transmission in brain circuits. Neuron 2012;76:98-115.

33 Nusbaum MP, Blitz DM, Marder E: Functional consequences of neuropeptide and smallmolecule co-transmission. Nat Rev Neurosci 2017;18:389-403.

34 Dougherty PM, Staats PS: Intrathecal drug therapy for chronic pain: from basic science to clinical practice. Anesthesiol 1999;91: 1891-1918. 